\title{
On inhibiting Auger intraband relaxation in InAs/GaAs quantum dot intermediate band solar cells
}

\author{
Stanko Tomić, ${ }^{1, a)}$ Antonio Martí, ${ }^{2}$ Elisa Antolín, ${ }^{2}$ and Antonio Luque ${ }^{2}$ \\ ${ }^{1}$ Joule Physics Laboratory, School of Computing, Science and Engineering, University of Salford, \\ Salford M5 4WT, United Kingdom \\ ${ }^{2}$ Instituto de Energía Solar, Universidad Politécnica de Madrid, Ciudad Universitaria sn, Madrid 28040, \\ Spain
}

(Received 18 May 2011; accepted 2 July 2011; published online 5 August 2011)

\begin{abstract}
The operation of ideal quantum dot intermediate band solar cell requires the largest possible reduction of carrier relaxation from the conduction band to the intermediate band (intraband relaxation) so that it approaches the radiative limit. In this respect, we examine the contribution to this relaxation of Auger related electron cooling non-radiative mechanisms and suggest ways of suppressing them. (C) 2011 American Institute of Physics. [doi:10.1063/1.3621876]
\end{abstract}

The intermediate band solar cell (IBSC) has been proposed as a means to improve efficiency over that of a single gap solar cell. ${ }^{1}$ The IBSC comprises the so called "intermediate band material," having an electronic band (intermediate band, IB) inside what otherwise would be a conventional semiconductor bandgap (Fig. 1). We will denote the total bandgap of the semiconductor as $E_{G}$, and its two parts, measured from the centre of the IB, as $E_{L}$ and $E_{H}$. To achieve its higher efficiency potential, the IB allows absorption of below-bandgap energy photons on transitions from the valence band $(\mathrm{VB})$ to the IB and from the IB to the conduction band $(\mathrm{CB})$. These absorption processes induce the corresponding carrier generation rates, denoted as $g_{I V}$ and $g_{C I}$ in Fig. 1, and these add up to the conventional carrier generation from the VB to the $\mathrm{CB}$ denoted as $g_{C V}$. Once carriers have been generated, they can also recombine. These recombination processes are denoted as $r_{X Y}$ for recombination taking place between bands $X$ and $Y$. For preserving the output voltage of the cell (equal to the difference of electron and hole quasi-Fermi levels, $e V=E_{F C}-E_{F V}$, where $e$ the electron charge $),{ }^{2}$ it is necessary that quasi-Fermi level separation exists between the $\mathrm{CB}$ quasi-Fermi level $\left(E_{F C}\right)$ and the IB quasi-Fermi level $\left(E_{F I}\right)$ and also between the VB quasiFermi level $\left(E_{F V}\right)$ and $E_{F I}$. These are increasingly difficult to achieve as the recombination rates $r_{I C}$ and $r_{I V}$, involving processes other than radiative recombination increase too.

Within the quantum dot (QD) approach, ${ }^{3}$ the IB is created from the confined electron states of the dots. ${ }^{4}$ The shape of the QD confinement potential is schematically indicated using dashed lines in the plot of the simplified bandgap diagram in Fig. 1. Several groups have manufactured QDIBSCs following this approach ${ }^{5-9}$ and experimentally demonstrated some of the principles of IBSC operation, such as the production of electron-hole pairs by below-bandgap energy photons ${ }^{10}$ or the existence of quasi-Fermi level separation between the $\mathrm{CB}$ and $\mathrm{IB} .{ }^{11}$ However, the experimental work carried out so far has also allowed identifying the factors that prevent boosting the efficiency of realistic QD-

\footnotetext{
a) Author to whom correspondence should be addressed. Electronic mail: s.tomic@salford.ac.uk.
}

IBSCs above that of single gap solar cells. ${ }^{12}$ Among the factors specific to the QD-IBSC approach, we point to the fast electron relaxation from $\mathrm{CB}$ to $\mathrm{IB}$, associated to the recombination process $r_{C I}$, which makes difficult maintaining the separation between $\mathrm{CB}$ and IB quasi-Fermi levels and, therefore, preserving the cell output voltage. The main mechanisms that increase $r_{C I}$ above its radiative limit, to be discussed next, are: (a) QD interface recombination, (b) energy transfer to lattice phonons, (c) interband Auger recombination, and (d) intraband-Auger recombination. For reference, the $\mathrm{CB}-\mathrm{IB}$ radiative decay rate is estimated to be around $130 \mathrm{~ns}$ in InAs/GaAs QDs. ${ }^{13}$ Following ShockleyVan Roosbroeck relation, ${ }^{14}$ this relatively large lifetime is the fundamental consequence of the weak absorption associated to the IB-CB transition. QD surface recombination

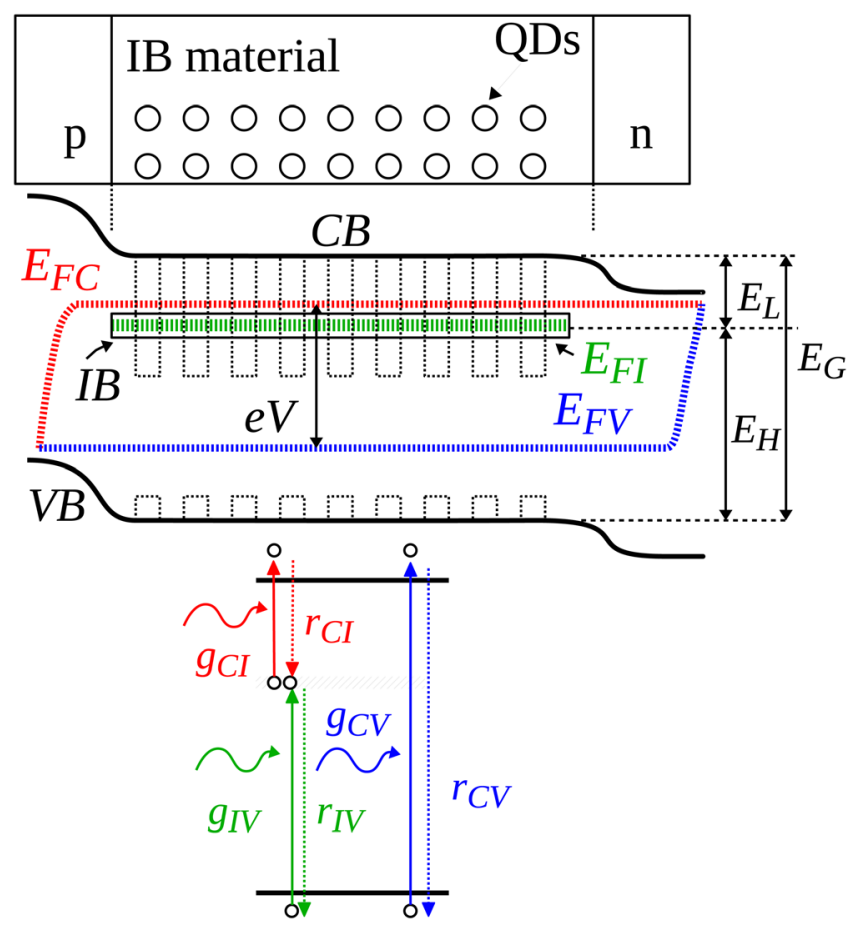

FIG. 1. (Color online) General structure of an intermediate band solar cell showing the simplified bandgap diagram and the generation recombination processes involved. 
(mechanism "a") can be diminished, in principle, when QDs are grown in the Stransky-Krastanov or Volmer-Weber mode since neither method leaves dangling bonds at the dotbarrier interface. ${ }^{15}$ Energy transfer to phonons (mechanism "b") is unlikely when $E_{L}$ is several times the energy of the lattice phonons (typically a few tens of meV). In fact, recent theoretical considerations ${ }^{13}$ suggest that the typical decay rate for this process is of the order of $\sim 20 \mathrm{~ns}$ for $E_{L} \approx 0.120$ $\mathrm{meV}$ in InAs/GaAs QDs. This is of the same order of magnitude as radiative recombination and would further increase if devices with larger $E_{L}$ are manufactured (as the IBSC concept demands).

In the interband Auger process, the energy of an electron in the $\mathrm{IB}$ recombining with a hole in the $\mathrm{VB}$ is transferred to another electron in the IB, which is promoted higher into the $\mathrm{CB}$, or to another hole in the $\mathrm{VB}$, which is promoted deeper into the VB. Taking again, as an example, the InAs/GaAs QD case, the average decay rate calculated for these processes is in the range $\sim 3-8$ ns. ${ }^{13}$ Although this decay rate can compete with the radiative mechanisms, it is not expected to be more detrimental for the IBSC performance than the conventional Auger recombination is in silicon solar cells. In particular, if QDs with an increased IB-CB absorption are engineered $^{16}$ so that the radiative decay time is decreased, the IB-VB Auger process will not have a major negative effect on IBSC efficiency.

In the InAs/GaAs, QDs electrons are thermalised to $e_{2}$ or $e_{1}$, i.e., the $p$-like orbitals, by fast electron-polaron relaxation before reaching the phonon bottleneck imposed by the energy split $E\left(e_{1}\right)-E\left(e_{0}\right)$ that is of the order of $\sim 100-150$ meV. Since the hole mass in InAs QDs is much larger than electron mass, confined hole states tend to be more densely spaced than electron states. A hole can come into $h_{0}$ state (top of VB) by fast hole-acoustic phonon thermalization. Both processes are on $p s$ time scale. In such a system, the energy transfer can occur when electron relaxes to its ground state $e_{0}$ (IB) and transfers the excess energy $\Delta E=E\left(e_{1}\right)-E\left(e_{0}\right)$ to the hole in its ground state $h_{0}$, exciting it deeper into VB. This is the intraband Auger non-radiative recombination or electron-cooling. To describe this process, we adopt a phenomenological formula for Auger decay rate derived from the standard time dependent perturbation theory and using Fermi's golden rule, ${ }^{17}$

$$
\frac{1}{\tau_{\mathrm{e}-\text { cool }}^{\mathrm{A}}}=\frac{2 \pi}{\hbar} \sum_{n} J\left(h_{0}, e_{1} ; h_{n}, e_{0}\right) \delta\left\{\Delta E-\left[E\left(h_{0}\right)-E\left(h_{n}\right)\right]\right\},
$$

where the Coulomb integral reads $J\left(h_{0}, e_{1} ; h_{n}, e_{0}\right)$ $\propto \int d \mathbf{r}_{1} \int d \mathbf{r}_{2} \psi_{h 0}^{*}\left(\mathbf{r}_{1}\right) \psi_{e 1}^{*}\left(\mathbf{r}_{2}\right) \psi_{h n}\left(\mathbf{r}_{1}\right) \psi_{e 0}\left(\mathbf{r}_{2}\right) /\left|\mathbf{r}_{1}-\mathbf{r}_{2}\right|, \quad$ and $\left\{\psi_{i}\right\}$ are the single-particle wave functions. The single-particle states are calculated using the 8 -band $\mathbf{k} \cdot \mathbf{p}$ Hamiltonian that takes into account the effects like band mixing, strain, and piezoelectric field, as implemented in the kppw code. ${ }^{18}$ In Eq. (1), we have used multiple final hole states $h_{n}$ where $n$ includes spin as well, since each final state might give some contribution to the Auger electron cooling rate. To take into account inhomogeneous line broadening due to size-distribution effects, as well as homogeneous line broadening, the $\delta$ function is replaced by a Gaussian $\exp \left[-(x / \sqrt{2} \Gamma)^{2}\right] /(\sqrt{2 \pi} \Gamma)$, defined by the phenomenological broadening $\Gamma=5 \mathrm{meV}$.

The typical decay rate associated to electron cooling process has been calculated to be in the range of ps (Ref. 13) (in very good agreement with other theoretical and experimental results ${ }^{19-22}$, and therefore, it is, in contrast to the cases discussed previously, the mechanism that could jeopardize the $E_{F C}-E_{F I}$ quasi-Fermi level separation and in turn the voltage preservation in the IBSC. If the excitonic gap between $e_{0}$ and $h_{0}$ (i.e., $E_{H}$ ) has to be preserved to maintain the good IBSC open circuit voltage, an alternative way to mediate the electron cooling rate is to reduce the overlap between the wave functions that enter Eq. (1). We further compare the Auger electron cooling times in realistic InAs/ GaAs QDs with those in an idealised structure in which the valence band offset (VBO) between InAs QD and GaAs barrier is set to zero, i.e., in VB confinement-less structure (Fig. 2). In Figure 3, we show the Auger electron cooling decay rates for (a) InAs/GaAs QDs and (b) the virtual InAs/GaAs QDs without confinement in the VB. By changing the QD base length from $b=15$ to $25 \mathrm{~nm}$, and keeping constant the aspect ratio between the base and height, $h$, in the pyramidal QDs to $b / h=2$, we have estimated that $\tau_{\mathrm{e}-\text { cool }}^{\mathrm{A}}$ for InAs/GaAs QD structures changes from 1 ps to $9.4 \mathrm{ps}$. In the same range of QD sizes for VB confinement-less structures, this time changes from $0.8 \mathrm{~ns}$ to $8.2 \mathrm{~ns}$, which represents an increase of three orders of magnitude when compared to the InAs/ GaAs QDs. This strong increase of $\tau_{\mathrm{e}-\text { cool }}^{\mathrm{A}}$ in structures of type (b) is attributed to the lack of any $\mathrm{VB}$ confinement. Lack of the VB confinement induces the strong hole delocalisation and in this way significantly reduces the values of Coulomb integrals $J$ [in Eq. (1)] and hence decreases the Auger electron cooling decay rate. Using week type-II heterostructures would bring about the same effect. However, IBSC based on the type-II QDs (Refs. 13 and 23) will reduce the absorption coefficient on VB-IB transitions as well. An optimal balance between those two effects, the desirable increase of electron cooling Auger time and the unwanted reduction of the absorption coefficient, is yet to be explored both theoretically and experimentally. The results for the Auger time constant are presented at the single particle level of theory, without full consideration of other electrons or holes in the system. ${ }^{17}$ Introducing a proper configuration interaction treatment of the correlation and exchange interaction with already existing particles involved in the Auger cooling process is likely to increase the Auger cooling time. (a)

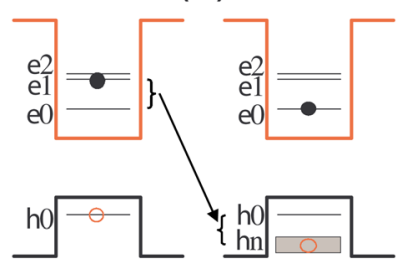

(b)

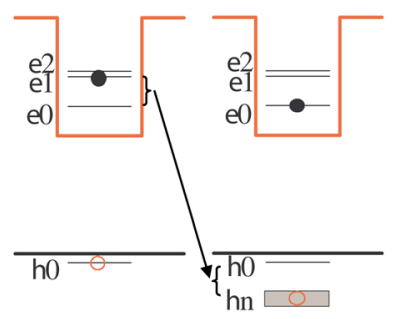

FIG. 2. (Color online) (a) Intraband Auger electron cooling recombination mechanisms in InAs/GaAs QDs and (b) intraband Auger electron cooling recombination mechanisms in virtual InAs/GaAs QDs with zero valence band offset. 


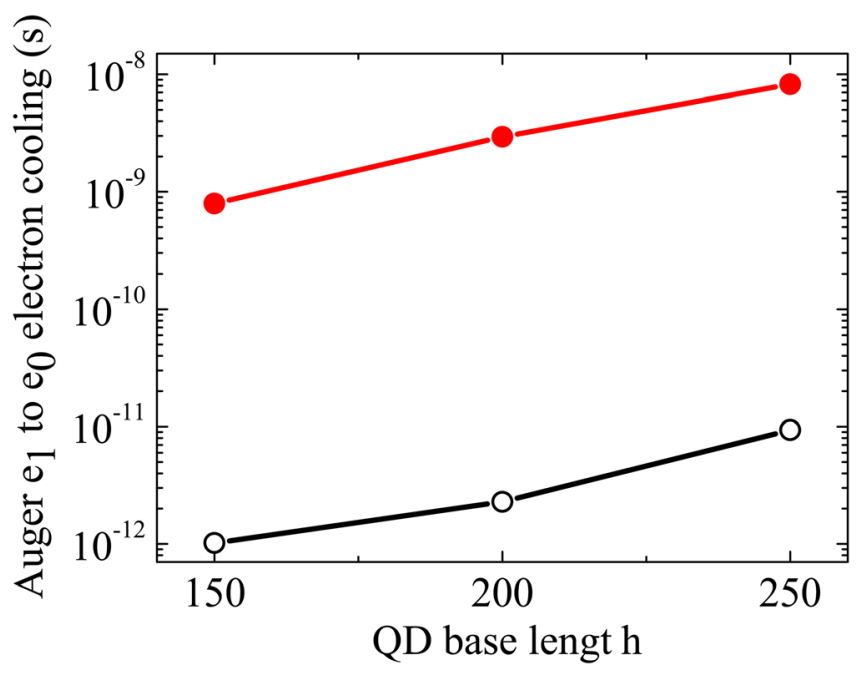

FIG. 3. (Color online) Auger electron cooling times as a function of the QD base length in InAs/GaAs QDs (open symbols) and in virtual InAs/GaAs QDs with zero valence band offset (solid symbols).

In InAs/GaAs QDs, with much weaker state confinement than in colloidal (for example, CdSe) QDs, the effects of correlation and exchange can only be weaker. The excitonic shift (i.e., $e_{0}-h_{0}$ Coulomb interaction energy) in InAs/GaAs QDs is only a few tens of meV (Ref. 24) One can therefore expect the many-body induced increase of Auger cooling time in VB confinement-less InAs/GaAs QDs to be relatively small.

In summary, we have shown theoretically that, with appropriate band structure engineering, it is possible to place the intraband Auger electron cooling decay timescale in the $n s$ range. Such an optimised design requires a VB confinement-less QD structure. The detrimental non-radiative decay rate in the InAs/GaAs QD based IBSC would then be increased by three orders of magnitude, i.e., in the same time range as other radiative processes.

S.T. thanks the UK's STFC e-Science SCARF facility for providing computer resources. A.M., E.A., and A.L. would like to acknowledge the funding of the Project DENQUIBAND (PLE2009-0045) by the Spanish Ministerio de Ciencia e Innovacion.

${ }^{1}$ A. Luque and A. Martí, Phys. Rev. Lett. 78, 5014 (1997).

${ }^{2}$ A. Luque and A. Martí, Prog. Photovolt.: Res. Appl. 9, 73 (2001).

${ }^{3}$ A. Martí, L. Cuadra, and A. Luque, in Conference Record of the 28th IEEE Photovoltaic Specialists Conference, IEEE, Anchorage (2000), p. 940.

${ }^{4}$ S. Tomić, T. S. Jones, and N. M. Harrison, Appl. Phys. Lett. 93, 263105 (2008).

${ }^{5}$ V. Popescu, G. Bester, M. C. Hanna, A. G. Norman, and A. Zunger, Phys. Rev. B 78, 205321 (2008).

${ }^{6}$ S. M. Hubbard, C. D. Cress, C. G. Bailey, R. P. Raffaelle, S. G. Bailey, and D. M. Wilt, Appl. Phys. Lett. 92, 123512 (2008).

${ }^{7}$ R. Oshima, A. Takata, and Y. Okada, Appl. Phys. Lett. 93, 083111 (2008). ${ }^{8}$ S. A. Blokhin, A. V. Sakharov, A. M. Nadtochy, A. S. Pauysov, M. V. Maximov, N. N. Ledentsov, A. R. Kovsh, S. S. Mikhrin, V. M. Lantratov, S. A. Mintairov, N. A. Kaluzhniy, and M. Z. Shvarts, Semiconductors 43, 514 (2009).

${ }^{9}$ D. Guimard, R. Morihara, D. Bordel, K. Tanabe, Y. Wakayama, M. Nishioka, and Y. Arakawa, Appl. Phys. Lett. 96, 203507 (2010).

${ }^{10}$ A. Marti, E. Antolin, C. R. Stanley, C. D. Farmer, N. Lopez, P. Diaz, E. Canovas, P. G. Linares, and A. Luque, Phys. Rev. Lett. 97, 247701 (2006).

${ }^{11}$ A. Luque, A. Marti, N. Lopez, E. Antolin, E. Canovas, C. Stanly, C. Farmer, L. Caballero, L. Caudra, and J. Balenzategui, Appl. Phys. Lett. 87, 083505 (2005).

${ }^{12}$ A. Luque and A. Marti, Adv. Mater. 22, 160 (2010).

${ }^{13}$ S. Tomić, Phys. Rev. B 82, 195321(2010).

${ }^{14}$ W. Van Roosbroeck and W. Shockley, Phys. Rev. 94, 1558 (1954).

${ }^{15}$ M. Sugawara, Self-Assembled InGaAs/GaAs Quantum Dots (Academic, Boston, 1999).

${ }^{16}$ A. Luque, A. Marti, E. Antolin, and P. Garcia-Linares, Sol. Energy Mater. Sol. Cells 94, 2032 (2010).

${ }^{17}$ L.-W. Wang, M. Califano, A. Zunger, and A. Franceschetti, Phys. Rev. Lett. 91, 056404 (2003).

${ }^{18}$ S. Tomić, A. G. Sunderland, and I. J. Bush, J. Mater. Chem. 16, 1963 (2006).

${ }^{19}$ G. A. Narvaez, G. Bester, and A. Zunger, Phys. Rev. B 74, 075403 (2006).

${ }^{20}$ T. S. Sosnowski, T. B. Norris, H. Jiang, J. Singh, K. Kamath, and P. Bhattacharya, Phys. Rev. B 57, R9423 (1998).

${ }^{21}$ T. Müller, F. F. Schrey, G. Strasser, and K. Unterrainer, Appl. Phys. Lett. 83, 3572 (2003).

${ }^{22}$ T. B. Norris, K. Kim, J. Urayama, Z. K. Wu, J. Singh, and P. K. Bhattacharya, J. Phys. D: Appl. Phys. 38, 2077 (2005).

${ }^{23}$ L. Cuadra, A. Marti, and A. Luque, Physica E 14, 162 (2002).

${ }^{24}$ N. Vukmirović and S. Tomić, J. Appl. Phys. 103, 103718 (2008). 